\title{
Characterizing areas of potential human exposure to eastern equine encephalitis virus using serological and clinical data from horses
}

\author{
J.-P. ROCHELEAU ${ }^{1 *}$, J. ARSENAULT ${ }^{1,2}$, N. H. OGDEN ${ }^{3}$, L. R. LINDSAY ${ }^{4}$, \\ M. DREBOT ${ }^{4}$ AND P. MICHEL ${ }^{1,3}$ \\ ${ }^{1}$ Groupe de recherche en épidémiologie des zoonoses et santé publique, Faculté de médecine vétérinaire, Université \\ de Montréal, Saint-Hyacinthe, Québec, Canada \\ ${ }^{2}$ Département de pathologie et microbiologie vétérinaire, Faculté de médecine vétérinaire, Université de Montréal, \\ Saint-Hyacinthe, Québec, Canada \\ ${ }^{3}$ Public Health Risk Science Division, Public Health Agency of Canada, National Microbiology Laboratory at \\ Saint-Hyacinthe, Québec, Canada \\ ${ }^{4}$ Public Health Agency of Canada, National Microbiology Laboratory, Zoonotic Diseases and Special Pathogens \\ Division, Winnipeg, Manitoba, Canada
}

Received 25 May 2016; Final revision 6 September 2016; Accepted 25 October 2016;

first published online 1 December 2016

\section{SUMMARY}

Eastern equine encephalitis (EEE) is a rare but severe emerging vector-borne disease affecting human and animal populations in the northeastern United States where it is endemic. Key knowledge gaps remain about the epidemiology of EEE virus (EEEV) in areas where its emergence has more recently been reported. In Eastern Canada, viral activity has been recorded in mosquitoes and horses throughout the 2000s but cases of EEEV in humans have not been reported so far. This study was designed to provide an assessment of possible EEEV human exposure by modelling environmental risk factors for EEEV in horses, identifying high-risk environments and mapping risk in the province of Quebec, Canada. According to logistic models, being located near wooded swamps was a risk factor for seropositivity or disease in horses [odds ratio (OR) $4 \cdot 15,95 \%$ confidence interval (CI) 1·16-14.8) whereas being located on agricultural lands was identified as protective (OR 0.75, 95\% CI 0.62-0.92). A better understanding of the environmental risk of exposure to EEEV in Canada provides veterinary and public health officials with enhanced means to more effectively monitor the emergence of this public health risk and design targeted surveillance and preventive measures.

Key words: Arboviruses, epidemiology, equine encephalitis (human), horse, risk assessment.

\section{INTRODUCTION}

Eastern equine encephalitis (EEE) is a vector-borne disease responsible for severe encephalitis in humans and horses. Case-fatality rates associated with clinical

\footnotetext{
* Author for correspondence: Dr J.-P. Rocheleau, Groupe de recherche en épidémiologie des zoonoses et santé publique, Faculté de médecine vétérinaire, Université de Montréal, 3200 rue Sicotte, Saint-Hyacinthe, Qc, Canada, J2S 2M2.

(Email: jean-philippe.rocheleau@umontreal.ca)
}

EEE neuroinvasive disease range from $35 \%$ to $75 \%$ in humans and can reach $90 \%$ in horses $[1,2]$. EEE virus (EEEV) has been considered endemic for decades in the eastern United States [3]. However, its geographical range seems to have expanded northwards recently with the first human cases of EEE reported in New Hampshire and Vermont in 2005 and 2012, respectively [1], increased and dispersed viral activity reported in Maine since 2009 [4] and sporadic outbreaks reported in horses from eastern Canada throughout 
the 2000s [5, 6]. Unprecedented numbers of equine clinical cases occurred yearly from 2008 to 2010 (total = 43) in the southern part of the province of Quebec, suggesting that the virus may have become endemic in this area [6]. In 2012, an equine EEEV serosurvey conducted in the same region revealed that more than $6 \%$ of horses had been infected with EEEV, which suggests that ecological niches (i.e. appropriate habitat, vectors and virus) exist to sustain EEEV transmission including potential spillover to human populations [7]. The characteristics of these ecological niches have, however, never been precisely identified. Studies conducted in northeastern United States may give some indications about the environmental characteristics of high-risk areas in eastern Canada. However, risk factors for EEEV spillover in affected areas of the United States were usually inferred from EEEV vector biology and feeding preferences $[8,9]$. Vector populations may change from one geographical area to another and the exact contribution of each vector species to EEEV spillover to humans still needs to be clarified $[10,11]$. Therefore, substantial knowledge gaps remain about risk to humans that can be applied to emerging areas such as Canada, particularly in areas where there is limited entomological surveillance directed towards EEEV as is currently the case in Quebec. A few pools of infected Culiseta melanura, the main enzootic vector of EEEV, were captured from a circumscribed wetland area of southern Quebec in 2009 and 2010 [12] but no further entomological investigations were conducted to assess the public health risk posed by EEEV in Quebec.

To address these knowledge gaps, the current study aimed to identify risk factors and map the risk of equine infection by EEEV in southern Quebec, Canada, using clinical and serological data from horses. We hypothesized that the level of EEEV activity is non-homogeneous across the region and assumed that the geographical distribution and the environmental factors linked to the risk in horses provide indirect indications of the risk distribution in humans.

\section{MATERIALS AND METHODS}

A dataset containing serological data on EEEV in horses was used for the analyses, alone and in combination with another one containing clinical outcomes of EEEV in horses. Logistic regression models were used for identifying environmental risk factors and a risk map was produced based on the output of the statistical models.

\section{Study area}

The study area consisted of five administrative regions from southern Quebec: Montérégie, Lanaudière, the Laurentides, Estrie and Centre-du-Québec (Fig. 1). These regions represent a mix of suburban, forested and agricultural lands with numerous wooded or non-wooded wetlands having the potential to sustain the EEEV enzootic transmission cycle [1, 3, 13-15]. The whole study area was divided in two zones: zone 1, which includes Montérégie, Lanaudière and the Laurentides, captured the location of 190 horses recruited in 2012 to the EEEV serological survey and 34 of the 43 equine EEE clinical cases that were reported from Quebec from 2008 to 2010; zone 2, which is adjacent to zone 1 , includes the regions of Estrie and Centre-du-Québec and the remaining nine equine EEE clinical cases reported from 2008 to 2010. Horses from zone 1 were used for building statistical models and horses from zone 2 were used to assess the sensitivity of the risk map (Fig. 1).

\section{Datasets}

The serosurvey dataset included 190 horses sampled in 2012 from 92 barns located within the study area. These horses had never been vaccinated against EEEV at the time of sampling, and 15 were seropositive to EEEV [7]. In addition to EEEV serological results, this dataset included information on horse characteristics (age, sex, breed), animal management (use of mosquito repellent, time spent outside daily, use of horse protective fly sheets or blankets), and characteristics of the barn in which they were housed (type of housing, use of window and door screens). The clinical cases' dataset included the 43 clinical equine EEE cases reported to the Ministry of Agriculture, Fisheries and Food of Quebec between 2008 and 2010. Individual characteristics of these 43 horses were not available. The two datasets included an accurate geolocation of the barns where each horse was being housed at the time of blood sampling or at the time of EEE diagnosis. With 34 of these 43 ill horses located within zone 1, a total sample size of 224 horses distributed over 62 municipalities was available for statistical modelling. The remaining nine ill horses, housed in six different barns, were distributed among six additional municipalities of zone 2 .

\section{Laboratory analyses}

Each serum sample from the serosurvey dataset was screened for antibodies to EEEV by a plaque 


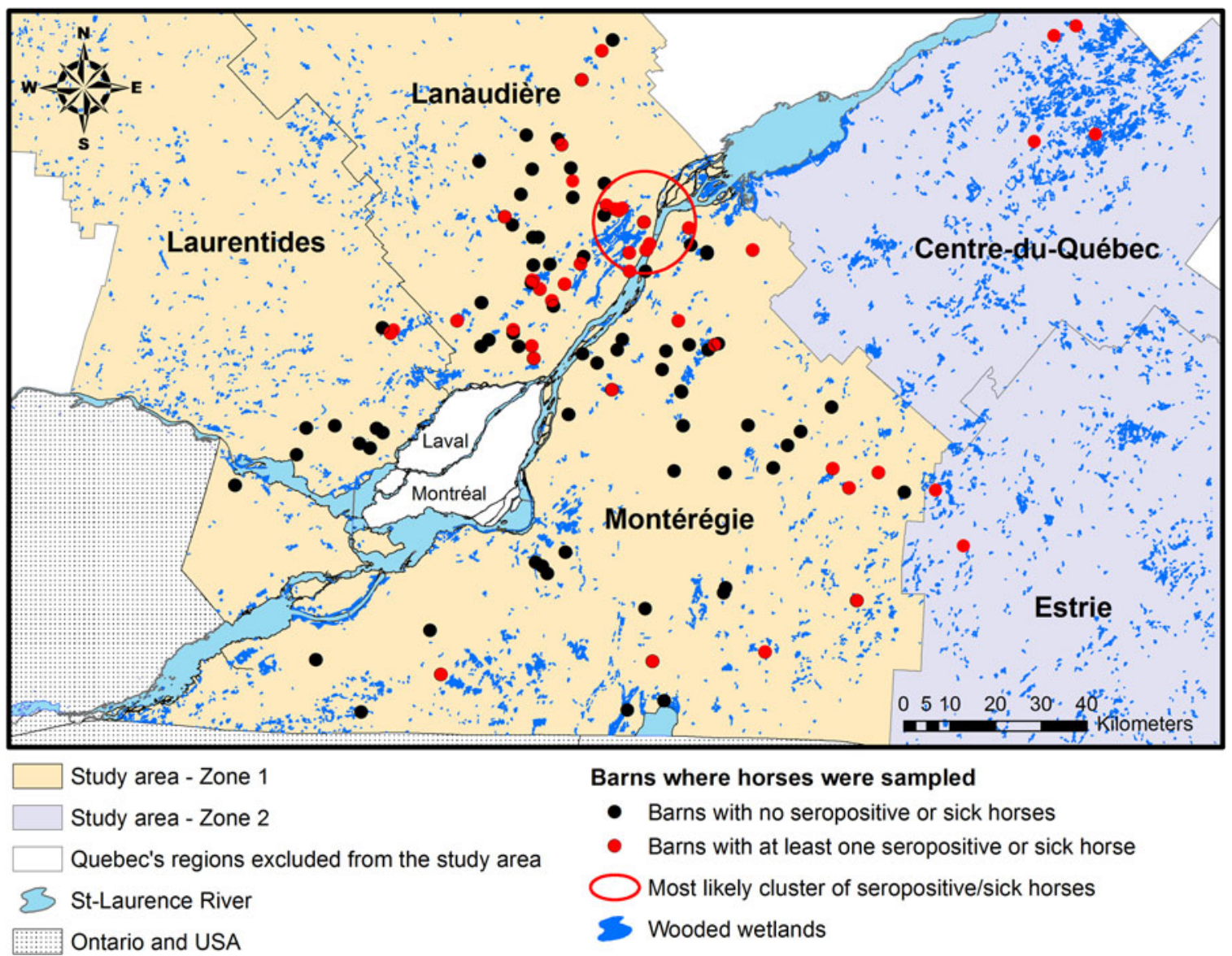

Fig. 1. Study area and geographical location of sampled or sick horses in the five selected administrative regions in southern Quebec, Canada.

reduction neutralization test (PRNT) at the National Microbiology Laboratory of the Public Health Agency of Canada as described previously [7]. Positive samples were titrated and samples were taken as positive when the PRNT neutralizing antibody titre was $\geqslant 1: 20$. Although the exact sensitivity and specificity of the PRNT was not specifically assessed in the current study, this serological assay is usually considered as a gold standard for arboviruses across species and is the recommended method for estimating prevalence of infection in the horse population according to the World Organization for Animal Health (OIE) [7, 16].

\section{Environmental data}

Land cover classification for the whole study area was retrieved from the Ecoforest Inventory System of the Ministry of Forests, Wildlife and Parks of Quebec (https://www.mffp.gouv.qc.ca/forets/inventaire/inventaire-quebec-meridional.jsp). For each horse, land cover variables were calculated as the proportion of the area covered either by hardwood, coniferous or mixed forest, developed, agricultural or dry bare land, orchards and surface water or wetlands inside a buffer of $5 \mathrm{~km}$ radius centred on the barn location. Land cover categories were defined according to the land cover classification system of the Fourth Eco-forest inventory of southern Quebec [17]. The buffer radius was chosen according to the average flying distance of Cs. melanura, the main enzootic vector of EEEV [3], ranging from 4 to $9 \mathrm{~km}$ according to previous studies $[18,19]$. Horses were assumed to spend most of their time in the close proximity to their barn.

\section{Statistical analyses}

Logistic regression analyses, using generalized estimating equations (GEE) with an exchangeable correlation structure (PROC GENMOD, SAS v. 9.4, SAS Institute Inc., USA) to account for potential clustering for horses living within the same municipality, were 
used to model the associations between the log odds of seropositivity or disease in horses and the aforementioned individual and environmental variables. Analyses were conducted first using the serosurvey dataset alone (model 1) to estimate associations between environmental variables and seropositivity while controlling for the potential confounding effects of individual factors. Analyses were then conducted on the combined serosurvey and EEEV clinical datasets for further identification of environmental risk factors on a larger sample including clinical outcomes. This sample was divided into two datasets: a training dataset including 190 horses $(85 \%$ of the horses from the dataset) that was used for model building (model 2) and a validation dataset including the remaining 34 horses that was used to assess the predictive power of model 2. Random allocation of the horses between the training and validation datasets was conducted using the SURVEYSELECT procedure in SAS v. 9.4. No validation dataset was produced for model 1 given the low number of positive outcomes in the serosurvey dataset. As no data on the individual characteristics of horses was available in the clinical cases database, model 2 only included environmental variables.

Unconditional associations between each environmental or individual factor and the outcome variable were estimated by univariate logistic regression. The assumption of a linear relationship between continuous predictors and the logit of the outcome was evaluated by plotting a loess smoothed curve of the predictor against the model's Pearson's residuals. If the relationship appeared not to be linear, the continuous predictor was categorized according to its quartiles and, when biologically relevant, adjacent categories that were not significantly different $(P<0.05)$ from each other were merged. All explanatory variables showing a significant unconditional association (using the criterion $P<0 \cdot 20$ ) were retained for inclusion in a multivariate model. Correlations between explanatory variables were evaluated using the Pearson correlation coefficient $(r)$ for continuous variables and the odds ratio (OR) obtained from simple logistic regression for categorical variables. When $r$ values were $>0.7$ or ORs were $>7$, one of the two correlated explanatory variables was chosen for further analysis [20] with selection on the basis of relevance to the biology of EEEV as reported in previous studies. The multivariate models were then refined by sequentially dropping explanatory variables whose removal did not significantly $(P>0.05)$ affect model deviance. An explanatory variable was considered as being a confounder if its removal from the model caused a $>20 \%$ change in the OR value of another explanatory variable. All confounders were forced into the final models. Two way interactions were tested between each of the final models' explanatory variables. In addition, for model 1 , the potential confounding effect of individual variables not selected from univariate analyses was assessed regardless of their $P$ value by forcing each of them one at a time in the final multivariate model. For the multivariate models, various correlation structures were compared based on QIC statistics (quasi-likelihood under the independence model criterion [21]). Outliers were identified by computing the standardized Pearson residuals and Cook's distances from the final multivariate model. Df-betas, which represent the variation in the parameter estimates when an observation is deleted from the dataset, were also computed for individual observations and for whole clusters (municipalities) to detect outliers at each of these levels. The predictive power of the multivariate models was assessed based on the area under the ROC curve (AUC) and the percentage of horses that were correctly classified by the models with a cut-off of $>0.5$ for classifying predicted values as positive. Predictive values for horses from the validation dataset were calculated based on the regression coefficients of model 2 and the predicted outcomes for these horses were compared with the observed outcomes. The AUC for model 2 was also computed based on the validation dataset.

A risk map was produced based on predicted values from the final logistic model. Model 2 was used based on its theoretically greater statistical power for environmental risk factor identification compared with model 1 . The study area was divided into a grid of $5 \times 5 \mathrm{~km}^{2}$ cells. Each cell's centroid was used as the centre of a $5-\mathrm{km}$ radius circular buffer. The percentage of the area inside each buffer that was represented by any of the environmental risk factors retained into the final logistic model was calculated and, for each buffer's centroid, the predicted value of the model was calculated [20]. A smoothed risk map was produced based on these predicted values by inverse distance weighting. The municipalities of Laval and Montreal, having a highly urbanized land cover unrepresentative of the municipalities where horses were sampled, were excluded from the risk map. Risk categories were set at very low, low, high and very high for predicted values ranging from 0.0 $0 \cdot 25,0 \cdot 25-0 \cdot 50,0 \cdot 50-0 \cdot 75$ and $0 \cdot 75-1 \cdot 00$, respectively. 
Table 1. Descriptive statistics and results from univariate logistic regression modelling EEEV serological status associated with individual variables using the serosurvey dataset (model 1)

\begin{tabular}{|c|c|c|c|c|c|}
\hline \multirow[b]{2}{*}{ Categorical predictors } & \multicolumn{2}{|l|}{ Count } & \multirow[b]{2}{*}{ OR } & \multirow[b]{2}{*}{$95 \% \mathrm{CI}$} & \multirow[b]{2}{*}{$P$ value } \\
\hline & Seropositive & Seronegative & & & \\
\hline \multicolumn{6}{|l|}{ Breed* } \\
\hline Arabian & 2 & 2 & $12 \cdot 0$ & $0 \cdot 86-168$ & 0.07 \\
\hline Other breed & 13 & 173 & Ref. & & \\
\hline \multicolumn{6}{|l|}{ Sex } \\
\hline Female & 6 & 89 & $0 \cdot 64$ & $0 \cdot 21-1 \cdot 91$ & $0 \cdot 42$ \\
\hline Male & 9 & 86 & Ref. & & \\
\hline \multicolumn{6}{|l|}{ Use of mosquito repellent } \\
\hline Never & 8 & 93 & Ref. & & \\
\hline Occasionally & 7 & 82 & $0 \cdot 85$ & $0 \cdot 23-3 \cdot 19$ & 0.82 \\
\hline \multicolumn{6}{|l|}{ Screens on doors and windows } \\
\hline Yes & 3 & 18 & Ref. & & \\
\hline No & 4 & 70 & $0 \cdot 98$ & $0 \cdot 13-7 \cdot 73$ & 0.99 \\
\hline n.a. (24 h outside daily) & 8 & 87 & & & \\
\hline \multicolumn{6}{|l|}{ Use of protective fly sheets } \\
\hline Never & 14 & 161 & Ref. & & \\
\hline \multirow[t]{2}{*}{ Occasionally } & 1 & 14 & $0 \cdot 46$ & $0 \cdot 007-31 \cdot 8$ & $0 \cdot 72$ \\
\hline & \multicolumn{2}{|l|}{ Mean } & & & \\
\hline Continuous predictors & Seropositive & Seronegative & OR & $95 \% \mathrm{CI}$ & $P$ value \\
\hline Time spent outside daily (hours) & $18 \cdot 3$ & $16 \cdot 1$ & $1 \cdot 06$ & $0 \cdot 95-1 \cdot 17$ & $0 \cdot 304$ \\
\hline Age (years) & $11 \cdot 9$ & $9 \cdot 8$ & $1 \cdot 05$ & $1 \cdot 00-1 \cdot 10$ & $0 \cdot 037$ \\
\hline
\end{tabular}

EEEV, Eastern equine encephalitis virus; OR, odds ratio (given by generalized estimating equations with an exchangeable correlation structure); CI, confidence interval; Ref., reference category; n.a.., not applicable.

* Ten breed categories were initially reported in the questionnaires. There was no statistically significant difference between breeds at $P<0 \cdot 20$ except for Arabian horses on univariate logistic regression.

The risk category that prevailed at the geographical location of each of the nine equine cases from the risk map validation dataset was assessed. Sensitivity of the risk map was assessed by comparing the geolocation of sick horses with the risk map categories using a cut-off of 0.5 for categorizing risk map areas as 'at risk' or 'not at risk'.

Possible geographical clusters of seropositive or sick horses were explored using the Kulldorff scan statistic (SaTScan v. 9.3.1) [22] and mapped in ArcGIS 10·2.2 (ESRI, USA). Scan statistics for high risk were computed on the combined clinical and serological datasets based on a Bernouilli model with the maximum cluster size representing $50 \%$ of the sample.

\section{Ethical standards}

Equine sampling for the serosurvey was approved by the Committee for ethical animal use of the Université de Montréal (certificate no. 12-Rech-1638).
The authors assert that all procedures contributing to this work comply with the ethical standards of the relevant national and institutional guides on the care and use of animals in research.

\section{RESULTS}

Descriptive statistics for individual risk factors in horses and environmental risk factors around sampled barns are presented in Tables 1 and 2. On average, barns were mainly surrounded by agricultural lands and forests. On average, wooded wetlands represented approximately $3 \%$ of the selected area around the barns.

Results from the univariate logistic regressions are presented in Table 1 (individual variables) and Table 3 (environmental variables). On univariate regression, agricultural land was the only statistically significant environmental risk factor in model 1 at $P<0 \cdot 05$, while agricultural land, wooded wetland and forested land were all statistically significant risk 
Table 2. Distribution of the percentages of land surface of land cover categories within a $5 \mathrm{~km}$ radius of barns where horses of the study were sampled

\begin{tabular}{llll}
\hline \hline & Percentile & & \\
\cline { 2 - 4 } Environmental characteristics (\%) & 25 & 50 & 75 \\
\hline Agricultural lands & $41 \cdot 3$ & $60 \cdot 0$ & $74 \cdot 9$ \\
Developed lands & $1 \cdot 6$ & $4 \cdot 5$ & $7 \cdot 4$ \\
Surface water & $0 \cdot 3$ & $0 \cdot 6$ & $1 \cdot 8$ \\
Hardwood forest (>75\% hardwood trees) & $10 \cdot 1$ & $14 \cdot 3$ & $20 \cdot 1$ \\
Mixed forest* (>25\% and <75\% of hardwood trees) & $2 \cdot 3$ & $5 \cdot 8$ & $13 \cdot 2$ \\
Coniferous forest (>75\% of conifers) & $0 \cdot 3$ & $1 \cdot 4$ & $3 \cdot 9$ \\
Total forest (mixed, hardwood and coniferous forests) & $14 \cdot 5$ & $22 \cdot 3$ & $40 \cdot 1$ \\
Dry bare land & $0 \cdot 1$ & $0 \cdot 3$ & $0 \cdot 9$ \\
Non-wooded wet lands & $0 \cdot 0$ & $0 \cdot 1$ & $1 \cdot 0$ \\
Wooded wet lands & $0 \cdot 7$ & $1 \cdot 9$ & $4 \cdot 6$ \\
Orchards & $0 \cdot 0$ & $0 \cdot 0$ & $0 \cdot 0$ \\
Other types of land & $0 \cdot 0$ & $0 \cdot 0$ & $0 \cdot 8$ \\
\hline \hline
\end{tabular}

* Mixed forest $=$ forested areas including $<75 \%$ of hardwood or coniferous trees.

factors at $P<0.05$ in model 2. Correlation analysis revealed that total forest was highly correlated with hardwood forest $(r=0.90)$ and mixed forest $(r=0 \cdot 88)$. The variable total forest was thus excluded from modelling. Results of the final multivariate logistic models are presented in Table 4. Agricultural lands were negatively associated with seropositivity in models 1 and 2 while wetlands were strongly and positively associated with seropositivity or disease in model 2 . None of the individual variables had a confounding effect on the other parameters of model 1. The lowest QIC was obtained by using an exchangeable correlation structure. With a cut-off at $>0.5$ for classifying a predicted value as a positive outcome, the final logistic models 1 and 2 correctly classified $77 \%$ and $79 \%$ of the observations of the equine datasets, respectively. The area under the ROC curve was 0.77 for both models, suggesting a fair predictive power. When used on the validation dataset, Model 2 correctly classified $91 \%$ of the horses from the validation dataset with an AUC of 0.75.

The analysis of DF-beta values in model 2 identified three municipalities from southern Lanaudière as having an unusual influence on the regression parameters. The analyses were thus recomputed while removing all horses from these three municipalities from the datasets. Variables retained in the final logistic model remained the same after removal of these influential observations with the OR increasing to $34 \%$ for wooded wetlands $(4 \cdot 15-5 \cdot 55, P=0 \cdot 043)$ and with no changes in the OR for agricultural lands $(0 \cdot 75-0 \cdot 75, P=0 \cdot 009)$ (results not shown).
The risk map based on the predicted values of logistic model 2 is presented in Figure 2. One of the nine equine clinical cases included in the risk map validation dataset occurred inside the model-defined very high-risk areas, three occurred inside a high-risk area, five occurred inside low-risk areas and none occurred inside very low-risk areas for an overall sensitivity of $44 \%$. Eight of the nine cases $(89 \%)$ occurred $<1.5 \mathrm{~km}$ from a high-risk area.

One statistically significant $(P<0 \cdot 001)$ cluster of seropositive or sick horses was detected with the Kulldorff scan statistic. This cluster had a radius of $11 \mathrm{~km}$ and was located over one of the largest wetlands in southern Quebec (Fig. 1).

\section{DISCUSSION}

Although EEEV viral activity has been relatively low in Quebec since the emergence of this virus in 2008, the current study allowed us to characterize EEE risk distribution over a large area of southern Quebec by using clinical and serological data from horses.

Wooded wetlands were strongly and positively associated with EEEV seropositivity or disease in horses. This is consistent with the ecology of the main vector of EEEV, Cs. melanura, which usually develops in wooded swamps with highly organic and shaded waters [23]. Moreover, a cluster of positive horses was detected within one of the largest wetlands in southern Quebec. Infected Cs. melanura were captured in this wetland in 2009 during an entomological study focused on EEEV in Quebec [12]. There is still some 
Table 3. Odds ratios and 95\% confidence intervals from univariate logistic regressions for environmental explanatory variables, models 1 and 2

\begin{tabular}{|c|c|c|c|c|c|c|}
\hline & \multicolumn{3}{|c|}{ Model 1: Serosurvey dataset alone $\dagger$} & \multicolumn{3}{|c|}{$\begin{array}{l}\text { Model 2: Serosurvey and clinical cases } \\
\text { dataset combined } ;\end{array}$} \\
\hline & OR & $95 \% \mathrm{CI}$ & $P$ value & OR & $95 \% \mathrm{CI}$ & $P$ value \\
\hline \multicolumn{7}{|l|}{ Continuous predictors* } \\
\hline Agricultural lands & $0 \cdot 77$ & $0 \cdot 61-0.99$ & $0 \cdot 04$ & $0 \cdot 73$ & $0 \cdot 59-0.91$ & $0 \cdot 004$ \\
\hline Developed lands & $1 \cdot 32$ & $0 \cdot 49-3 \cdot 50$ & $0 \cdot 58$ & $0 \cdot 94$ & $0 \cdot 44-2 \cdot 00$ & $0 \cdot 87$ \\
\hline Wooded wet lands & $2 \cdot 70$ & $0 \cdot 89-8 \cdot 17$ & $0 \cdot 08$ & $5 \cdot 20$ & $1 \cdot 63-16 \cdot 6$ & $0 \cdot 005$ \\
\hline Non-wooded wetlands & $3 \cdot 85$ & $0 \cdot 09-163$ & $0 \cdot 48$ & $10 \cdot 7$ & $0 \cdot 43-269 \cdot 8$ & $0 \cdot 15$ \\
\hline Surface water & $1 \cdot 03$ & $0 \cdot 55-1 \cdot 93$ & $0 \cdot 93$ & $1 \cdot 14$ & $0 \cdot 53-2 \cdot 44$ & $0 \cdot 74$ \\
\hline Dry bare land & $14 \cdot 7$ & $0 \cdot 64-338$ & $0 \cdot 09$ & $6 \cdot 39$ & $0 \cdot 11-380 \cdot 6$ & $0 \cdot 37$ \\
\hline \multicolumn{7}{|l|}{ Categorized predictors } \\
\hline \multicolumn{7}{|l|}{ Hardwood forest } \\
\hline$<14 \cdot 3 \%$ & Ref. & & & Ref. & & \\
\hline$\geqslant 14 \cdot 3 \%$ & $3 \cdot 18$ & $0 \cdot 92-11 \cdot 0$ & $0 \cdot 07$ & $4 \cdot 43$ & $1 \cdot 87-10 \cdot 5$ & $0 \cdot 0007$ \\
\hline \multicolumn{7}{|l|}{ Mixed forest } \\
\hline$<5 \cdot 8$ & Ref. & & & Ref. & & \\
\hline $5 \cdot 8-13 \cdot 2$ & $2 \cdot 23$ & $0 \cdot 41-12 \cdot 2$ & $0 \cdot 35$ & $4 \cdot 16$ & $1 \cdot 37-12 \cdot 7$ & $0 \cdot 012$ \\
\hline$\geqslant 13 \cdot 2$ & $2 \cdot 95$ & $0 \cdot 61-14 \cdot 3$ & $0 \cdot 18$ & $5 \cdot 87$ & $1 \cdot 78-19 \cdot 3$ & $0 \cdot 004$ \\
\hline \multicolumn{7}{|l|}{ Coniferous forest } \\
\hline$<3 \cdot 9$ & Ref. & & & Ref. & & \\
\hline$\geqslant 3.9$ & $1 \cdot 50$ & $0 \cdot 46-4 \cdot 84$ & $0 \cdot 50$ & $2 \cdot 13$ & $1 \cdot 07-4 \cdot 24$ & 0.032 \\
\hline \multicolumn{7}{|l|}{ Orchards } \\
\hline$<0 \cdot 16$ & Ref. & & & Ref. & & \\
\hline$\geqslant 0 \cdot 16$ & $0 \cdot 39$ & $0 \cdot 35-6 \cdot 20$ & $0 \cdot 60$ & $0 \cdot 34$ & $0 \cdot 10-1 \cdot 15$ & $0 \cdot 08$ \\
\hline
\end{tabular}

OR, Odds ratio; CI, confidence interval; Ref., reference category.

* OR presented for an increase of $10 \%$ of the area represented by the selected land cover within a buffer of $5 \mathrm{~km}$ radius around the barn.

$\dagger$ Based on serosurvey database, 2012 ( $n=190$ horses).

$\$$ Merged data from the serosurvey and the clinical cases database ( $n=224$ horses).

uncertainty about the contribution of other vector species in the transmission of EEEV, some studies suggesting that Cs. melanura would be both the epidemic and enzootic vector [10], while others suggesting that bridge vectors (that feed on birds and large mammals) are generally needed for the spillover of the virus to occur $[11,24]$. Nevertheless, the current study underlines the important impact of living near ecosystems supporting EEEV enzootic cycles on the risk in horses and, it would be expected, humans. The wide geographical distribution of both equine cases and wooded wetlands throughout southern Quebec (Fig. 1) suggests that many areas of Quebec could sustain EEEV transmission. The mechanisms that could drive such a geographical dispersion, however, remain unclear. The only region of Quebec where equine EEE clinical cases were repeatedly reported from 2008 to 2010 was southern Lanaudière. All clinical cases detected in the other regions were reported in only 2008 with no cases subsequently. Thus, the mere presence of wetlands does not seem to be sufficient for EEE equine cases to occur from year to year in a given region and EEEV may need to be reintroduced yearly in most geographical areas for viral amplification to occur. Alternatively, EEEV may need to amplify within bird populations to a level sufficient for spill-over to horses, and conditions that would support this amplification may not occur on an annual basis. The phenomenon of recurrence of EEE three years in a row in southern Lanaudière would warrant further investigations on the potential mechanisms driving EEEV endemicity. The potential for transovarial transmission of EEEV in mosquito vectors is not well supported in the literature $[14,25,26]$ and, although a number of authors have hypothesized that EEEV could overwinter in various vertebrate hosts [27-29], the overwintering mechanism of EEEV have not been clearly elucidated.

Along with wetlands, agricultural lands had a significant influence on the estimated risk. Given the natural breeding ecosystems of Cs. melanura, it would be 
Table 4. Odds ratio and 95\% confidence intervals for the final multivariate logistic models predicting EEEV serological status or disease in horses according to individual and environmental variables

\begin{tabular}{llll}
\hline \hline & OR & $95 \%$ CI & $P$ value \\
\hline Model 1 (Serosurvey dataset alone) & & & \\
$\quad$ Agricultural lands* & $0 \cdot 73$ & $0.58-0.92$ & 0.008 \\
$\quad$ Breed (Arabian vs. others) & $22 \cdot 0$ & $1 \cdot 01-474$ & 0.049 \\
Model 2 (Serosurvey and clinical cases dataset combined) & & & \\
$\quad$ Agricultural lands* & $0 \cdot 75$ & $0.62-0.92$ & 0.006 \\
$\quad$ Wooded wet lands* & $4 \cdot 15$ & $1 \cdot 16-14 \cdot 8$ & $0 \cdot 028$ \\
\hline \hline
\end{tabular}

EEEV, Eastern equine encephalitis virus; OR, odds ratio; CI, confidence interval.

* OR presented for an increase of $10 \%$ of the area represented by the selected land cover within a buffer of $5 \mathrm{~km}$ radius around the barn.

biologically plausible that agricultural lands, which are usually drained, could act as barriers against the expansion of EEEV enzootic cycle outside of wetland areas. However, other vector species such as Aedes vexans, which feeds frequently on horses and which has sometimes been identified as a potential bridge vector for EEEV [30], can be very abundant in agricultural areas of southern Quebec [31]. The protective effect of agricultural lands suggests that these vectors would have a limited ability to contribute to EEEV dispersal. Limited competency of vector species that breed in agricultural lands and limited dispersal of viraemic avian hosts outside wetland areas during summer could possibly contribute to decreased risk of spillover of EEEV far away from wetlands. These findings support the hypothesis that Cs. melanura would contribute to progressive dispersal of EEEV from one swamp focus to other adjacent swamp foci during the summer season [14].

Breed was identified as a potential individual risk factor in the multivariate model based on the serosurvey dataset with a p-value slightly under the selected cut-off, Arabian horses having higher odds of being seropositive. However, the dataset included only two seropositive and two seronegative Arabian horses which may not be representative of the population of Arabian horses from Quebec. To our knowledge, no breed predilection has been reported previously for EEE in horses. However, most epidemiological studies on EEE in horses focused on environmental factors or management practices [9] and breed predilection for EEE may not have been reported. Further studies would be needed to more thoroughly assess the potential association between breed and the risk of exposure to EEEV or EEE clinical disease in horses.

Overall, multivariate models had a fair predictive power. With a cut-off at 0.5 for the dichotomous classification of risk areas, the risk map had a relatively weak sensitivity for correctly predicting areas where the nine sick horses were located from the risk map validation dataset. However, the fact that $89 \%$ of the sick horses from the risk map validation dataset lived $<1.5 \mathrm{~km}$ away from a high-risk area suggested that, overall, the risk map had some potential for detecting areas where EEEV risk might occur. A number of factors may explain that sick horses were mainly found at the periphery of high-risk areas. Given that swamp areas are usually less suitable for the development of built environments, lower horse population densities and, consequently, lower counts of reported equine cases is expected from the centre of high-risk areas. Moreover, infected vectors can fly from wetland areas towards hosts living in areas dominated by other types of land covers, contributing to EEEV dispersal away from swamp foci. Finally, horses' movements around their barns could have led to the virus being contracted a few kilometres away from the geographical locations used in this study. A more rigorous assessment of risk map validity would have required to know the geographical distribution of the equine 'at risk' population, data that are not currently available in Quebec. The total equine population in the study area was estimated to 11000 horses in 2007. However, according to a previous study [7], approximately $80-90 \%$ of this population would be vaccinated against EEEV, reducing the at risk population to approximately 2000 horses. The low total count of clinical cases of EEE in horses, which may be partly explained by the vaccination coverage, also limited our capacity to further evaluate the predicted risk map. Nevertheless, the risk map presented here may provide guidance for further surveillance and research in southern Quebec and represents a first step in the understanding of the geographical 


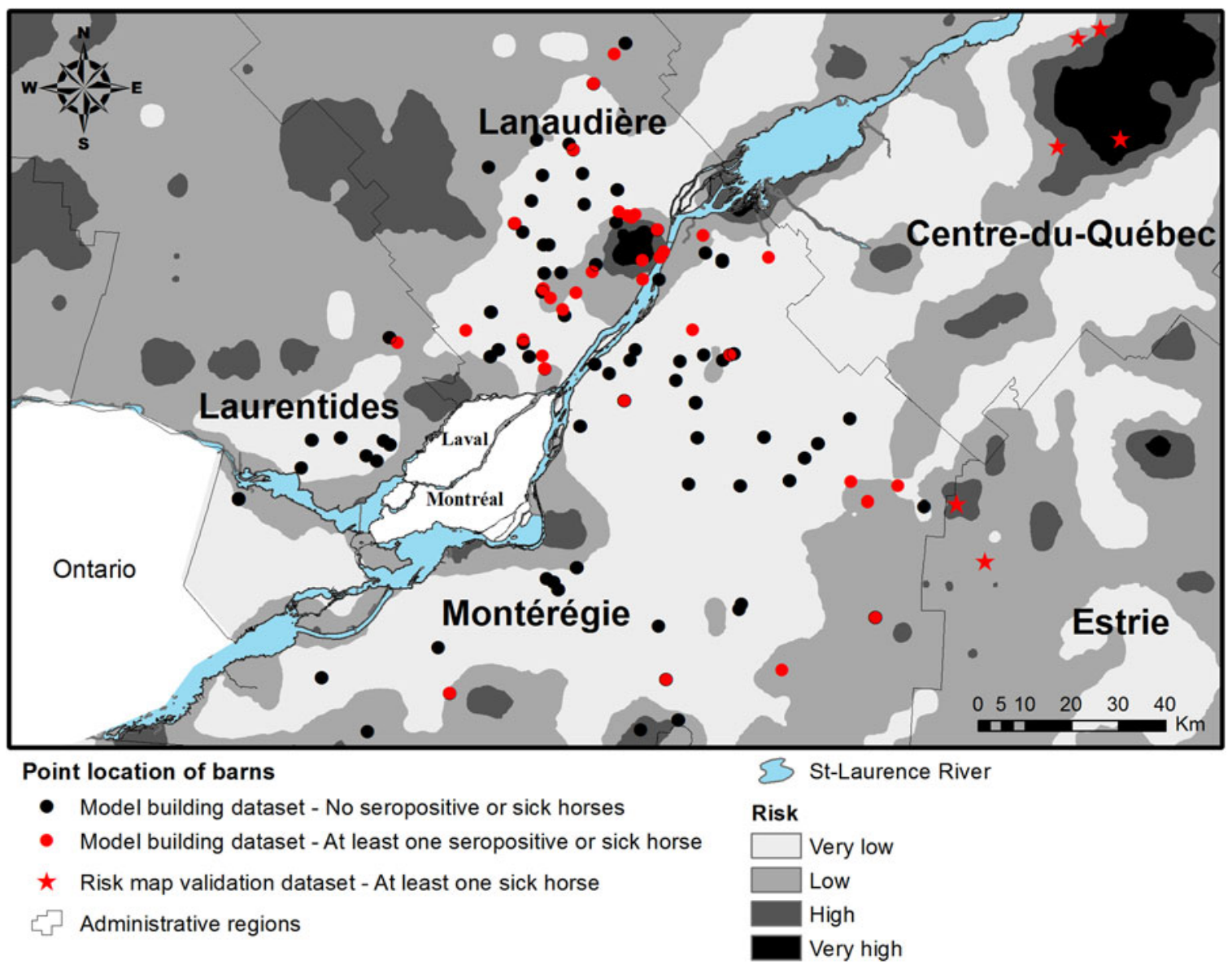

Fig. 2. Geographical risk distribution for eastern equine encephalitis virus in horses as predictive by the final multivariate logistic regression model.

distribution of risk. Given that all clinical cases of EEE in horses from Quebec were reported between the months of July and October and that climatic conditions do not allow for vector activity in Quebec during winter, the reported risk distribution refers temporally to the July to November period. Risk is expected to be low during spring and null during winter.

Differences were observed among the univariate associations estimated from the seroprevalence dataset alone and from the combined serological and clinical datasets. These differences outlined some methodological issues which go beyond the simple lack of statistical power. One of these issues is related to the potential effect of misclassification of environmental variables: as the neutralizing EEEV antibodies (e.g. IgG) detected by PRNT are thought to be long-lasting in horses [2] and as horses are often relocated from one barn to the other [7], the exact time and geographical location of exposure is uncertain in seropositive horses and this misclassification can potentially bias associations towards the null. With acute equine EEEV clinical cases, which have an incubation period of a few days [2], there is a high probability that infection occurred close to where the horse lived at the time of diagnosis thus limiting misclassification of exposure. This phenomenon may explain why the strength of the associations and the ability to detect statistically significant associations were higher in model 2 compared to model 1 .

These models brought out new epidemiological knowledge that could be relevant to public health. Although the study area did not include the most densely populated areas of southern Quebec, villages and small towns can be found throughout each of the high-risk areas identified in the current study. Thus, there seems to be a real risk of human exposure in these areas. Given that disease or seropositivity in horses represent EEEV spillover out of the enzootic cycle to accidental hosts, the environmental risk factors identified in horses likely represent environments where spillover to humans may also occur. 
Confirming and characterizing risk of EEEV exposure in humans in highly populated areas such as Montreal would, however, require the use of other surveillance tools, such as entomological surveillance or the use of a different or complementary sentinel animal.

This study illustrates an approach to indirectly infer risk of EEEV exposure in humans from specific epidemiological information derived from the environment, therefore building grounds for public health prevention strategies. Risk factors outlined in this study seem consistent with the human risk factors previously reported in the northeastern United States. Further studies are needed to validate if risk areas are the same in horses and humans and to improve our understanding of the ecological pathways leading from EEEV introduction to virus amplification and finally to spillover of EEEV to accidental hosts in Canada. Quantitative and spatial analyses of animal epidemiological data can bring new insights about infection dynamics for rare or emerging arboviral diseases in a public health perspective. To our knowledge, this study is the first to characterize and map risk of EEEV exposure in Canada in the absence of systematic entomological surveillance. The approach could allow for increased preparedness for EEEV outbreaks and other emerging vector-borne diseases by targeting surveillance and planning interventions.

\section{ACKNOWLEDGEMENTS}

We thank all participating veterinary practitioners for their help in equine sampling and veterinarians of the Ministry of Agriculture, Fisheries and Food of Quebec for collecting data on equine clinical cases. This study was funded by the Public Health Agency of Canada.

\section{DECLARATION OF INTEREST}

None.

\section{REFERENCES}

1. Armstrong PM, Andreadis TG. Eastern equine encephalitis virus - old enemy, new threat. New England Journal of Medicine 2013; 368: 1670-1673.

2. Gibbs EP, Long MT. Equine alphaviruses. In: Sellon DC, Long MT, eds. Equine Infectious Diseases. St Louis: Saunders Elsevier, 2014, pp. 210-217.
3. Calisher CH. Medically important arboviruses of the United States and Canada. Clinical Microbiology Reviews 1994; 7: 89-116.

4. Gibney KB, et al. Eastern equine encephalitis: an emerging arboviral disease threat, Maine, 2009. Vector Borne and Zoonotic Diseases 2011; 11: 637-639.

5. Ontario Ministry of Agriculture Food and Rural Affairs. Equine neurological disease (http://omafra.gov.on.ca/ english/livestock/horses/westnile.htm). Accessed 13 April 2016.

6. Quebec Ministry of Agriculture Fisheries and Food. Eastern equine encephalomyelitis [in French] (http:// www.mapaq.gouv.qc.ca/fr/Productions/santeanimale/mal adies/transmissibleshumain/Pages/encephalomyeliteequin edelest.aspx). Accessed 5 June 2015.

7. Rocheleau JP, et al. Eastern equine encephalitis virus: high seroprevalence in horses from Southern Quebec, Canada, 2012. Vector Borne and Zoonotic Diseases 2013; 13: 712-718.

8. Centers for Disease Control and Prevention. Eastern equine encephalitis (http://www.cdc.gov/EasternEquine Encephalitis/index.html). Accessed 7 July 2012.

9. United States Department of Agriculture. Epidemiology and ecology of eastern equine encephalomyelitis (http://www.aphis.usda.gov/animal_health/emergingissues/ downloads/EEE042004.pdf). Accessed 7 June 2015.

10. Armstrong PM, Andreadis TG. Eastern equine encephalitis virus in mosquitoes and their role as bridge vectors. Emerging Infectious Diseases 2010; 16: 1869-1874.

11. Hassan HK, et al. Avian host preference by vectors of eastern equine encephalomyelitis virus. American Journal of Tropical Medicine and Hygiene 2003; 69: 641-647.

12. Vincent $\mathbf{C}$, et al. Surveillance of eastern equine encephalomyelitis in Quebec [in French]. 2010.

13. Emord DE, Morris CD. Epizootiology of eastern equine encephalomyelitis virus in upstate New York, USA. VI. Antibody prevalence in wild birds during an interepizootic period. Journal of Medical Entomology 1984; 21: 395-404.

14. Howard JJ, et al. Evidence for multiple foci of eastern equine encephalitis virus (Togaviridae: Alphavirus) in central New York State. Journal of Medical Entomology 1996; 33: 421-432.

15. Ducks unlimited. Wetlands: a source of life - portrait of wetlands [in French], 2007.

16. World Organisation for Animal Health. Manual of Diagnostic Tests and Vaccines for Terrestrial Animals 2015 (http://www.oie.int/international-standard-setting/ terrestrial-manual/). Accessed 15 April 2016.

17. Ministry of Forests Wildlife and Parks of Quebec. Ecoforest stratification standards-Fourth eco-forest inventory of southern Quebec [in French], 2013, pp. 7-11.

18. Howard JJ, White DJ, Muller SL. Mark-recapture studies on the Culiseta (Diptera, Culicidae) vectors of eastern equine encephalitis-virus. Journal of Medical Entomology 1989; 26: 190-199.

19. Estep LK, et al. Estimation of dispersal distances of Culex erraticus in a focus of eastern equine encephalitis 
virus in the southeastern United States. Journal of Medical Entomology 2010; 47: 977-986.

20. Dohoo IR, Martin SW, Stryhn H. Veterinary Epidemiologic Research, 2nd edn. Charlottetown, PEI: VER, Inc., 2009.

21. Pan W. Akaike's information criterion in generalized estimating equations. Biometrics 2001; 57: 120-125.

22. Kulldorff M. A spatial scan statistic. Communications in Statistics-Theory and Methods 1997; 26: 1481-1496.

23. Morris CD. Eastern equine encephalomyelitis. In: Monath T, ed. Arboviruses: Epidemiology and Ecology. Boca Raton: CRC Press, 1988, pp. 1-20.

24. Molaei G, et al. Dynamics of vector-host interactions in avian communities in four eastern equine encephalitis virus foci in the Northeastern U.S. PLoS Neglected Tropical Diseases 2016; 10: e0004347.

25. Morris CD, Srihongse S. An evaluation of the hypothesis of transovarial transmission of eastern equine encephalomyelitis virus by Culiseta melanura. American Journal of Tropical Medicine and Hygiene 1978; 27: $1246-1250$.
26. Weaver SC, Scott TW, Lorenz LH. Patterns of eastern equine encephalomyelitis virus infection in Culiseta melanura (Diptera: Culicidae). Journal of Medical Entomology 1990; 27: 878-891.

27. Owen JC, et al. Test of recrudescence hypothesis for overwintering of eastern equine encephalomyelitis virus in gray catbirds. Journal of Medical Entomology 2011; 48: 896-903.

28. White G, et al. Competency of reptiles and amphibians for eastern equine encephalitis virus. American Journal of Tropical Medicine and Hygiene 2011; 85: 421-425.

29. Bingham AM, et al. Detection of eastern equine encephalomyelitis virus RNA in North American snakes. American Journal of Tropical Medicine and Hygiene 2012; 87: 1140-1144.

30. Cupp EW, et al. Transmission of eastern equine encephalomyelitis virus in central Alabama. American Journal of Tropical Medicine and Hygiene 2003; 68: 495-500.

31. Maire A, Aubin A. Mosquitoes from Quebec (Diptera: Culicidae) ecological synthesis study [in French]. Memoirs of the Entomological Society of Quebec 1980; 6: 21-22. 